\title{
Effectiveness of health education as an intervention designed to prevent female genital mutilation/cutting (FGM/C): a systematic review
}

Susan Waigwa ${ }^{1 *}$, Lucy Doos², Caroline Bradbury-Jones ${ }^{1}$ and Julie Taylor ${ }^{3}$

\begin{abstract}
Background: Female Genital Mutilation/Cutting (FGM/C) is a harmful practice that violates the human rights of women and girls. Despite global efforts to restrict the practice, there have been few reports on major positive changes to the problem. Health education interventions have been successful in preventing various health conditions and promoting service use. They have also been regarded as promising interventions for preventing FGM/C. The objective of this systematic review is to synthesise findings of studies about effectiveness of health education as an intervention to prevent FGM/C.
\end{abstract}

Methods: The electronic databases searched were MEDLINE, EMBASE, Cochrane library, Web of Science, Psych INFO, CINAHL and ASSIA. Our search included papers published in the English language without date limits. Study quality was assessed using the Mixed Methods Appraisal Tool (MMAT). A predesigned data recording form was used to extract data from the included studies which were summarised by comparing similar themes.

Results: Twelve out of 359 individual studies met our inclusion criteria. Seven studies were quantitative, three were qualitative and two used mixed methods. Six studies tested before and after the interventions, four studies assessed the effectiveness of previous interventions used by different research teams and two studies endorsed the intervention. Four main factors emerged and were associated with facilitating or hindering the effectiveness of health education interventions: sociodemographic factors; socioeconomic factors; traditions and beliefs; and intervention strategy, structure and delivery.

Conclusions: It is vital to target factors associated with facilitating or hindering the effectiveness of health education for FGM/C. This increases the possibility of effective, collective change in behaviour and attitude which leads to the sustainable prevention of FGM/C and ultimately the improved reproductive health and well-being of individuals and communities.

Keywords: Community, 'Circumcision, Female', Prevention, Female genital mutilation, Health education

\footnotetext{
* Correspondence: smw554@bham.ac.uk

${ }^{1}$ School of Nursing, College of Medical and Dental Science, University of

Birmingham, Edgbaston, Birmingham B15 2TT, UK

Full list of author information is available at the end of the article
}

C The Author(s). 2018 Open Access This article is distributed under the terms of the Creative Commons Attribution 4.0 International License (http://creativecommons.org/licenses/by/4.0/), which permits unrestricted use, distribution, and reproduction in any medium, provided you give appropriate credit to the original author(s) and the source, provide a link to the Creative Commons license, and indicate if changes were made. The Creative Commons Public Domain Dedication waiver (http://creativecommons.org/publicdomain/zero/1.0/) applies to the data made available in this article, unless otherwise stated. 


\section{Plain English summary}

Female genital mutilation (FGM/C) is a harmful practice that involves total or partial removal of female genitalia without medical purpose. It is mainly practised in some countries in Africa, Asia, the Middle East and some communities in South America. Migration, however, has been associated with the wide spread of FGM/C around the globe. It is performed on young girls and causes short-term and life-long consequences for women as well as extended consequences for families and the community at large. These consequences increase burden to the health systems. Health education interventions are among the prominent forms of interventions that can prevent the practice of FGM/C. However, its impact is dependent on factors that facilitate or hinder effectiveness. Our review revealed that these factors include sociodemographic factors; socioeconomic factors; traditions and beliefs; and intervention strategy, structure and delivery. To ensure the effectiveness of health education interventions, these factors should be considered.

In conclusion, health education interventions have the potential to prevent FGM/C. They can produce a sustainable impact on the reproductive health and wellbeing of individuals as well as communities. The findings from this study imply that, with caution, health education interventions that focus on FGM/C can be effectively implemented in different populations.

\section{Background}

Female genital mutilation/cutting (FGM/C) is a violation against the human rights of women and children such as the right to freedom from discrimination, torture and violence; the right to health; and the right to education. FGM/C involves the total or partial removal of female genitalia without medical purpose. The global prevalence of FGM/C among girls and women is estimated to be over 200 million. It is concentrated particularly in some African, Asian and Middle Eastern countries [1-3]. However, migration has been associated with the wide spread of FGM/C around the globe [4]. Records from 2012 estimate that about 513,000 girls and women had either undergone or were at risk of $\mathrm{FGM} / \mathrm{C}$ in the United States of America (USA) [5, 6]. In 2015, England and Wales, in the United Kingdom (UK), recorded 137,000 girls and women subjected to FGM/C and 60,000 girls at risk [7].

The World Health Organization (WHO) classifies FGM/C into four types; Type I- Clitoridectomy; Type IIExcision; Type III- Infibulation; and Type IV- Other procedures, including piercing and incising. The practice has been associated with adverse short-term health consequences such as heavy bleeding and tetanus infections; and long-term consequences such as recurring vaginal and pelvic infections, menstrual complications, difficulties during pregnancy and childbirth; and psychological problems such as Post-Traumatic Stress Disorder (PTSD), anxiety and depression [8-11].

FGM/C, which is usually performed on young girls between the age of infancy and 15 years [12], has no medical benefits and medical professionals around the globe are prohibited from carrying out the practice $[4,13]$. Whilst the Universal Declaration of Human Rights as well as other global conventions and declarations emphatically oppose the practice [14]. There are a number of factors that allow FGM/C to continue. These include cultural/traditional factors, which are tied up with rituals and complex belief systems [15], religious factors, which are enforced by specific religious beliefs and teachings [16], and health/hygiene factors, which include myths associated with perceived health benefits [17].

The attempts to deal with the negative consequences of FGM/C have unfortunately developed into the medicalisation of the procedure, whereby guarantees of safety are erroneously proffered as a reason for FGM/C to be carried out by health professionals. However, the engagement of health professionals in such procedures inevitably cause harm and constitutes a violation of medical conduct $[4,13,18]$. Consequently, greater effort has been made to deter health professionals from engaging in FGM/C by legal consequences of the practice. However, despite the global efforts to curb FGM/C, there has been few reports on major positive changes of the problem [1].

Health education is the main intervention of interest in this review. It involves different learning experiences designed to help individuals and communities improve their health by increasing their knowledge or influencing their attitude [19]. This goes beyond sharing or disseminating information about a health issue to address motivation, skills, confidence, and communication of information. Differences in economic, social and environmental conditions; individual risk factors and behaviours; and use of health systems are also considered [20].

It is vital for health education interventions to aim at long-term changes to the health behaviour and the norms that are attributed to a health problem. However, evaluation of the effectiveness of interventions depend on documenting the outcomes, effects, formation, process, cost-effectiveness and benefits of the interventions [20].

Health education programmes have been effective in addressing various health related issues such as smoking uptake and cessation, healthy pregnancy and improved newborn outcomes [21-23]. Health education has also succeded in promoting the use of services such as family planning, particularly in communities that are reluctant to access such services [24]. It has also been considered to be a potential intervention for preventing $\mathrm{FGM} / \mathrm{C}$. 
There are some studies which have reported successful health education interventions in preventing FGM/C globally, but there is need for more exploration of the interventions including their effects in different communities [25]. To our knowledge, there is no systematic review that has synthesised the evidence and ensured understanding of the effectiveness of health education interventions as discrete interventions for FGM/C. The purpose of this review therefore, was to explore the effectiveness of health education as an intervention to prevent $\mathrm{FGM} / \mathrm{C}$ in the affected communities.

\section{Methods}

We searched electronic databases for published work using comprehensive search strategies. Seven main international databases were systematically searched. These included; MEDLINE, EMBASE, Cochrane library, Web of Science, Psych INFO, CINAH and ASSIA. These databases were selected to best represent source material in health, applied health, and human science. Grey literature was also searched and the reference lists from included studies and systematic reviews about FGM/C interventions were hand searched. Search terms were structured carefully to include the problem, intervention comparator and expected outcomes (using a PICO formulation). The terms included female genital mutilation OR female circumcis* OR female genital cutting, affected communit", health educat* AND/OR health literacy, prevent* OR abandon* OR eliminat* OR stop*(see Additional file 1). English articles with no date restriction were searched. The search was completed in June 2016. Endnote $\times 7$ was utilised as the main reference manager.

The first author (SW) screened titles independently and a second reviewer (HS) independently repeated the process to ensure no relevant studies were excluded. The same reviewers independently decided on the full texts to be included by scrutinising the abstracts. Predetermined inclusion and exclusion criteria were used to guide the screening and selection process.

We included studies focusing on communities affected by FGM/C. There was no limit to the population by geographical location. The included studies either used or discussed health education as an intervention. They had a purpose of disseminating information to individuals or groups of people with an aim of preventing FGM/C as the primary outcome. We considered all study designs with no defined publication timeframe. We excluded studies that did not focus on communities affected by FGM/C and those that focused on medical or cosmetic procedures like vulvectomy or labiaplasty. Studies with a focus on other interventions and studies involving circumcision/genital cutting other than female genital cutting, for instance male circumcision were also excluded.
Data from included studies were extracted using a predesigned data recording form, including general details of the study, intervention description, study outcomes and conclusions. Data were recorded on Microsoft Excel software, which the team used to crosscheck extraction details and ensure accuracy. Discrepancies were discussed and agreed upon within the review team.

Study quality was assessed using the Mixed Methods Appraisal Tool (MMAT) Version 2011 [26]. This was chosen because of its ability to review mixed method studies alongside qualitative and quantitative studies in a single combined tool.

The included papers did not have data that were suitable for meta-analysis, similar to Yang et al. [27], due to heterogeneity. We therefore carried out a thematic analysis focusing on the main themes that were evident in the included studies. Both manifest and latent themes were explored and described as understood by the authors.

\section{Results}

The search elicited 359 publications from which 12 full text articles met our inclusion criteria, as shown in the PRISMA flow chart (Figure 1).

The populations involved in the 12 included studies were from Africa and the Middle East, where FGM/C is prevalent. Half of the studies (six) tested knowledge about FGM/C before and after intervention [28, 29, 30, $31,32,33]$. Four different studies assessed the effectiveness of previous interventions used by different research teams $[34,35,36,37]$. The remaining two studies endorsed the intervention as a solution for preventing FGM/C following evidence of the relevance of health education interventions to communities affected by FGM/C [38, 39].

From the 12 included studies, seven were quantitative, three qualitative and two used mixed methods. The quality mean-score, of all included studies was $83.33 \%$, which was above the predetermined cut-off mark of $50 \%$, as measured with the Mixed Method Assessment Tool (MMAT). All of the included studies scored above $50 \%$ in each of their respective categories and therefore qualified for inclusion. Table 1 presents a detailed summary of each of the 12 included studies.

\section{Factors affecting the effectiveness of health education}

Four major themes were identified: sociodemographic factors; socioeconomic factors; traditions and beliefs; and intervention strategy, structure and delivery. These are described further in this section in a nonlinear process. All the studies highlighted at least two themes and discussed their contribution in either enabling or hindering the effectiveness of health education intervention. A summary of the themes is presented in Table 2. 


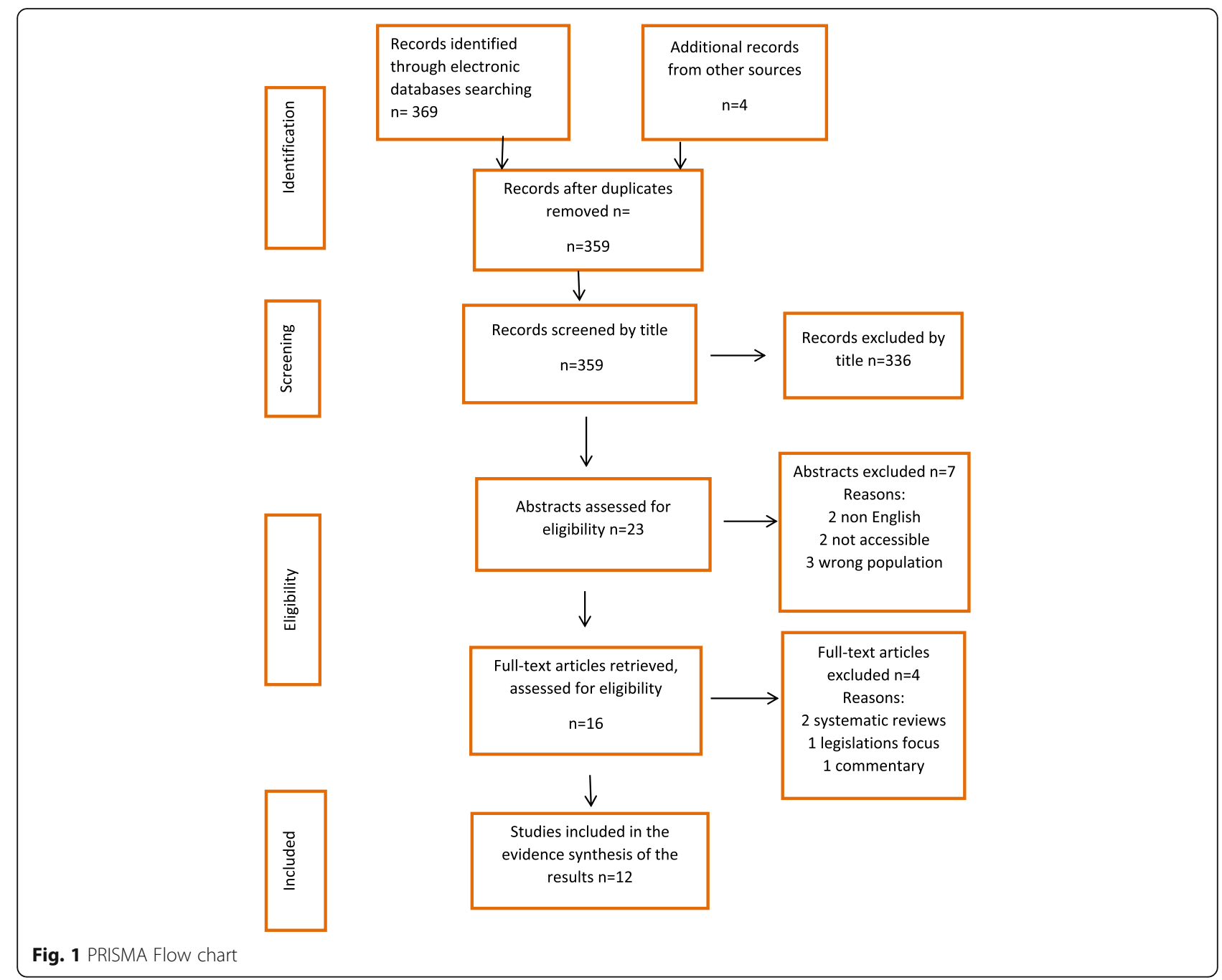

\section{Sociodemographic factors}

Six sociodemographic elements were described in the included studies. They include age, ethnicity, language, gender, marital status and residential status.

\section{Age}

Two of the twelve studies reported that the age of the populations involved in health education interventions influenced the effectiveness of the interventions. In general, younger populations were more amenable to the interventions. In a study by Olaitan [36] with parents in Nigeria, knowledge of older parents was found to be significantly different from that of younger parents. In the same vein, Alo and Gbadebo [39] concluded that among populations that approved the practice, the levels of FGM/C prevalence were higher among older respondents. They suggested this was because younger respondents were more likely to be school educated, which increased their chances of engaging with FGM/C health education that encouraged abandonment of the practice.

\section{Ethnicity}

Three studies reported that ethnic differences between facilitators and communities sometimes influenced the effectiveness of health education interventions due to backlash. It was concluded that facilitators and interviewers needed to belong to the same ethnic groups as participants. Partnering with communities prevented a top-down approach that enhanced the effectiveness of the interventions $[28,30,31]$. If facilitators and interviewers were of different ethnicity from that of the participants, they were required to familiarise themselves with the cultural and structural customs by integrating with the communities beforehand $[28,31]$.

\section{Language}

Two studies highlighted the potential of language barriers reducing the effectiveness of health education interventions [32, 35]. Facilitators struggled with delivering messages and participants also found it hard to understand the messages which were not in their local 
Table 1 Summary of included papers

\begin{tabular}{|c|c|c|c|c|c|c|c|}
\hline Author and year & $\begin{array}{l}\text { Setting and } \\
\text { prevalence }\end{array}$ & Population & $\begin{array}{l}\text { Sample type and } \\
\text { recruitment } \\
\text { strategy }\end{array}$ & $\begin{array}{l}\text { Study design } \\
\text { and } \\
\text { comparison } \\
\text { group }\end{array}$ & $\begin{array}{l}\text { Method and } \\
\text { quality of } \\
\text { studies }\end{array}$ & $\begin{array}{l}\text { Information/activities } \\
\text { intervention offered/ } \\
\text { evaluated }\end{array}$ & Outcome/results \\
\hline $\begin{array}{l}\text { Ajuwon J } \\
\text { Ademola, } \\
\text { Brieger R } \\
\text { William, } \\
\text { Oladepo } \\
\text { Oladimeji, deniyi } \\
\text { D Joshua (1995) }\end{array}$ & $\begin{array}{l}\text { South West } \\
\text { Nigeria } \\
\text { FGM/C Type I }\end{array}$ & $\begin{array}{l}\text { Male } \\
\text { practitioners } \\
\text { Males and } \\
\text { females } \\
\text { community } \\
\text { leaders } \\
\text { Males and } \\
\text { females in } \\
\text { focus } \\
\text { groups } \\
\text { Nigeria, } \\
\text { Yoruba } \\
\text { community }\end{array}$ & $\begin{array}{l}\text { Male and female } \\
\text { community leaders } \\
\text { Married and } \\
\text { unmarried men } \\
\text { and women } \\
\text { Practitioners/ } \\
\text { circumcisers } \\
\text { Leaders in } \\
\text { community } \\
\text { Community } \\
\text { members } \\
\text { Practitioners age } \\
45 \text { and } 70 \text { years } \\
\text { Community } \\
\text { leaders and focus } \\
\text { groups, not } \\
\text { specified }\end{array}$ & Qualitative & $\begin{array}{l}\text { Interviews } \\
75 \%\end{array}$ & $\begin{array}{l}\text { General knowledge } \\
\text { about FGM/C }\end{array}$ & $\begin{array}{l}\text { There was high need } \\
\text { for health education } \\
\text { interventions especially } \\
\text { for indigenous } \\
\text { surgeries }\end{array}$ \\
\hline $\begin{array}{l}\text { Allam MF, } \\
\text { Irala-Esteves DJ, } \\
\text { Navajas FCR, } \\
\text { Castillo DSA, } \\
\text { Hoashi JS, } \\
\text { Pankovich MB, } \\
\text { Liceaga RJ. } \\
\text { (2001) }\end{array}$ & $\begin{array}{l}\text { Universities } \\
\text { in Cairo, } \\
\text { Egypt }\end{array}$ & $\begin{array}{l}\text { Males and } \\
\text { females } \\
\text { Egypt }\end{array}$ & $\begin{array}{l}\text { University students } \\
\text { mean age of } \\
20.97 \text { years of } \\
\text { medical and } \\
20.73 \text { years from } \\
\text { non-medical } \\
\text { Belong to a } \\
\text { community that } \\
\text { practice FGM }\end{array}$ & $\begin{array}{l}\text { Cross- } \\
\text { sectional } \\
\text { No } \\
\text { comparison } \\
\text { group }\end{array}$ & $\begin{array}{l}\text { Face-to-face } \\
\text { interviews } \\
32 \text {-item } \\
\text { questionnaire } \\
100 \%\end{array}$ & $\begin{array}{l}\text { General information } \\
\text { about FGM }\end{array}$ & $\begin{array}{l}\text { High proportion } \\
\text { considered discussions } \\
\text { in the media to play } \\
\text { an important role in } \\
\text { banning of FGM/C } \\
\text { People are aware of } \\
\text { the dangers involved, } \\
\text { are more likely to be } \\
\text { against the practice }\end{array}$ \\
\hline $\begin{array}{l}\text { Alo \& Gbadebo, } \\
\text { (2011) }\end{array}$ & $\begin{array}{l}\text { Southwest } \\
\text { Nigeria }\end{array}$ & $\begin{array}{l}\text { Women } \\
\text { Southwest } \\
\text { Nigeria }\end{array}$ & $\begin{array}{l}\text { Women who have } \\
\text { at least one living } \\
\text { daughter } \\
\text { Belong to a } \\
\text { community } \\
\text { affected by FGM/C } \\
15-49 \text { years }\end{array}$ & Survey & $\begin{array}{l}\text { Interviews } \\
50 \%\end{array}$ & $\begin{array}{l}\text { General knowledge } \\
\text { about FGM/C }\end{array}$ & $\begin{array}{l}\text { Respondents with } \\
\text { post-secondary educa- } \\
\text { tion were at least likely } \\
\text { to have their daughters } \\
\text { cut. } \\
\text { Only } 52 \% \text { of the } \\
\text { women were aware of } \\
\text { the health hazards } \\
\text { associated with FGC } \\
\text { Participants from high } \\
\text { socio-economic status } \\
\text { are least likely to have } \\
\text { their daughters cut }\end{array}$ \\
\hline $\begin{array}{l}\text { Asekun- } \\
\text { Olarinmoye } \\
\text { EO,Amusan OA } \\
(2008)\end{array}$ & $\begin{array}{l}\text { Shao } \\
\text { community } \\
\text { is in Kwara } \\
\text { State Nigeria } \\
\text { Between } 60 \\
\text { and } 70 \% \\
\text { FGM/C Type } \\
\text { I and II }\end{array}$ & $\begin{array}{l}\text { Males and } \\
\text { females } \\
\text { Yoruba, } \\
\text { Nigeria }\end{array}$ & $\begin{array}{l}\text { Residents of Shao } \\
\text { town above } \\
10 \text { years } \\
\text { Participants } \\
\text { belonged to a } \\
\text { community that } \\
\text { practice FGM/C } \\
\text { Modal age of } 30- \\
39 \text { and } 20-29 \text { in } \\
\text { pre-intervention } \\
\text { and post interven- } \\
\text { tion respectively }\end{array}$ & survey & $\begin{array}{l}\text { Questionnaires } \\
100 \%\end{array}$ & $\begin{array}{l}\text { Health talks in } \\
\text { vernacular on female } \\
\text { genital anatomy, } \\
\text { nature and types of } \\
\text { FGM/C,complications, } \\
\text { beliefs that encourage } \\
\text { it } \\
\text { Pictures were utilised } \\
\text { to illustrate female } \\
\text { genitalia, different } \\
\text { types of mutilation } \\
\text { Questions and answer } \\
\text { sessions utilised for } \\
\text { further discussions }\end{array}$ & $\begin{array}{l}\text { No statistical } \\
\text { significance difference } \\
\text { between the } \\
\text { composition and } \\
\text { socio-demographic } \\
\text { characteristics } \\
\text { Education status, age } \\
\text { and gender were } \\
\text { found to be statistically } \\
\text { significant in } \\
\text { association to those } \\
\text { who had their } \\
\text { daughters excised } \\
\text { Positive impact of the } \\
\text { health education } \\
\text { intervention on the } \\
\text { attitude of the } \\
\text { respondents to FGM/C } \\
\text { and intentions to } \\
\text { subject their daughters }\end{array}$ \\
\hline
\end{tabular}


Table 1 Summary of included papers (Continued)

\begin{tabular}{|c|c|c|c|c|c|c|c|}
\hline Awuah JB (2008) & $\begin{array}{l}\text { Aboabo } \\
\text { No.1 - } \\
\text { Suburb of } \\
\text { Kumasi } \\
75-85 \% \\
\text { prevalence } \\
\text { (24.5\% of } \\
\text { women) } \\
\text { FGM/C Type } \\
\text { ॥ }\end{array}$ & $\begin{array}{l}\text { Females } \\
\text { African- } \\
\text { Ghana }\end{array}$ & $\begin{array}{l}\text { Those whose } \\
\text { address contacts } \\
\text { could be traced to } \\
\text { their homes } \\
\text { Participants belong } \\
\text { to a community } \\
\text { that practice FGM } \\
\text { No indicated age }\end{array}$ & $\begin{array}{l}\text { Exploratory } \\
\text { research }\end{array}$ & $\begin{array}{l}\text { Face to face } \\
\text { interviews } \\
75 \%\end{array}$ & $\begin{array}{l}\text { Background, } \\
\text { knowledge and } \\
\text { experiences of being } \\
\text { circumcised and } \\
\text { facing birth } \\
\text { complications } \\
\text { participant } \\
\text { Suggestions of ways } \\
\text { to prevent FGM/C } \\
\text { from the participants }\end{array}$ & $\begin{array}{l}43 \% \text { suggested health } \\
\text { professionals should } \\
\text { use health talks } \\
14 \% \text { suggested use of } \\
\text { dramas and role plays } \\
\text { by health educators } \\
14 \% \text { believe that } \\
\text { education of females } \\
\text { would help } \\
4 \% \text { thought use of } \\
\text { mass media to educate } \\
\text { the public would help }\end{array}$ \\
\hline $\begin{array}{l}\text { Babalola } \\
\text { S,Brasington A, } \\
\text { Agbasimalo A, } \\
\text { Helland A, } \\
\text { Nwanguma E, } \\
\text { Onah N. (2006) }\end{array}$ & $\begin{array}{l}\text { Enugu state: } \\
3 \text { local } \\
\text { government } \\
\text { areas; i. Uzo- } \\
\text { Uwani,ii. Isi- } \\
\text { Uzo and iii. } \\
\text { Enugu South } \\
\text { FGC } \\
\text { prevalence of } \\
\text { Enugu -59\% } \\
\text { Ebonyi -78\% } \\
\text { Usually type } \\
\text { I and II of } \\
\text { FGC are } \\
\text { performed }\end{array}$ & $\begin{array}{l}\text { Males and } \\
\text { females } \\
\text { Africa-Enugu } \\
\text { and Ebonyi } \\
\text { states, } \\
\text { Nigeria }\end{array}$ & $\begin{array}{l}\text { Participants belong } \\
\text { to communities } \\
\text { affected by FGC } \\
\text { a. Enugu state for } \\
\text { intervention } \\
\text { b.Ebonyi state for } \\
\text { comparison c. age } \\
18 \text { to } 59 \mathrm{~d} \text {. men } \\
\text { and women }\end{array}$ & $\begin{array}{l}\text { cross- } \\
\text { sectional } \\
\text { survey } \\
\text { Ebonyi state } \\
\text { for } \\
\text { comparison }\end{array}$ & $\begin{array}{l}\text { Interviews } \\
100 \%\end{array}$ & $\begin{array}{l}\text { Examination of } \\
\text { knowledge, attitudes } \\
\text { and practices } \\
\text { surrounding women's } \\
\text { reproductive health } \\
\text { Support and training } \\
\text { in development of } \\
\text { action plan } \\
\text { Discussions on social } \\
\text { and health } \\
\text { complications of FGC }\end{array}$ & $\begin{array}{l}\text { Increased knowledge } \\
\text { leading to widespread } \\
\text { intentions not to practice } \\
\text { Increased self-efficacy } \\
\text { to refuse pressure to } \\
\text { perform } \\
\text { Extremely religious } \\
\text { people are less likely to } \\
\text { abandon FGC } \\
\text { Large number of } \\
\text { children was associated } \\
\text { with intentions not to } \\
\text { practice }\end{array}$ \\
\hline $\begin{array}{l}\text { Chege J, Askew } \\
\text { I, Igras S, Mutesh } \\
\text { JK. (2004) }\end{array}$ & $\begin{array}{l}\text { Semi-arid } \\
\text { rural in } \\
\text { Ethiopia and } \\
\text { Kenya } \\
\text { Ethiopia- } \\
\text { Awash } \\
\text { Woredea. } \\
\text { Kenya-lfo in } \\
\text { Dadaab } \\
76 \% \text { (Ethiopia) } \\
34 \% \text { (Kenya) } \\
\text { Specific } \\
\text { community: } \\
\text { 91\% } \\
\text { (Ethiopia) } \\
\text { 100\% } \\
\text { (Kenya) } \\
\text { FGM/C Type } \\
\text { III }\end{array}$ & $\begin{array}{l}\text { Males and } \\
\text { females } \\
\text { African- } \\
\text { Ethiopians } \\
\text { and Somali } \\
\text { in Kenya }\end{array}$ & $\begin{array}{l}\text { Participants must } \\
\text { have experienced } \\
\text { or lived with } \\
\text { people who have } \\
\text { experienced FGM } \\
\text { Ethiopia-8 to } \\
60 \text { years } \\
\text { Kenya-15 to } \\
60 \text { years } \\
\text { Participants belong } \\
\text { to communities } \\
\text { affected by FGC }\end{array}$ & $\begin{array}{l}\text { Quasi- } \\
\text { experimental } \\
\text { Ethiopia-six } \\
\text { villages in } \\
\text { Amibara } \\
\text { Woreda. } \\
\text { Kenya- } \\
\text { Hagadera } \\
\text { camp }\end{array}$ & $\begin{array}{l}\text { Interviews } \\
75 \%\end{array}$ & $\begin{array}{l}\text { Community level } \\
\text { education outreach } \\
\text { activities using } \\
\text { behaviour- } \\
\text { communication- } \\
\text { change } \\
\text { Community level } \\
\text { advocacy } \\
\text { Training dispensary } \\
\text { service providers in } \\
\text { treating complications } \\
\text { and counselling } \\
\text { clients on FGC related } \\
\text { areas }\end{array}$ & $\begin{array}{l}\text { Percentage of those } \\
\text { who support } \\
\text { abandonment in } \\
\text { Ethiopia intervention } \\
\text { group increased by } \\
32 \% \text {-control group } \\
\text { increased by } 10 \% \text { - } \\
\text { Kenya-intervention } \\
\text { group remained at } \\
23 \% \text {-comparison group } \\
\text { increased by } 8 \% \\
\text { Percentage of those who } \\
\text { do not intend to cut - } \\
\text { Ethiopia intervention } \\
\text { group increased by } 26 \%- \\
\text { control reduced by } 1 \%- \\
\text { Kenya intervention group } \\
\text { increased by } 3 \%- \\
\text { comparison increased by } \\
8 \% \\
\text { Lower levels of } \\
\text { exposure to FGC } \\
\text { information translates } \\
\text { to lower increases in } \\
\text { positive attitudes and } \\
\text { intent behaviours. }\end{array}$ \\
\hline $\begin{array}{l}\text { Diop NJ, Askew } \\
\text { I (2009 }\end{array}$ & $\begin{array}{l}\text { Kolda } \\
\text { Region in } \\
\text { Southern } \\
\text { Senegal } \\
94 \% \\
\text { prevalence } \\
\text { FGM/C Type } \\
\text { I and II }\end{array}$ & $\begin{array}{l}\text { Men and } \\
\text { women } \\
\text { Senegal }\end{array}$ & $\begin{array}{l}\text { Males and females } \\
\text { from villages } \\
\text { where TOSTAN } \\
\text { programme had } \\
\text { been implemented } \\
\text { and in Older than } \\
15 \text { years }\end{array}$ & $\begin{array}{l}\text { Survey Quasi- } \\
\text { experimental, } \\
\text { pre-and post- } \\
\text { intervention } \\
\text { longitudinal } \\
\text { design } \\
\text { Comparison- } \\
\text { villages } \\
\text { where the } \\
\text { programme } \\
\text { had not } \\
\text { reached }\end{array}$ & $\begin{array}{l}\text { Interviews } \\
100 \%\end{array}$ & $\begin{array}{l}\text { Modules about: } \\
\text { Human rights, } \\
\text { Problem-solving } \\
\text { process, Basic hygiene } \\
\text { and Women's health }\end{array}$ & $\begin{array}{l}\text { Statistically significant } \\
\text { differences in the } \\
\text { proportion of girls } \\
\text { reported to have been } \\
\text { cut in intervention } \\
\text { group } \\
\text { Significant attitudinal } \\
\text { and behavioural } \\
\text { changes leading to } \\
\text { mass declaration } \\
\text { against FGM/C } \\
\text { Education, facilitated } \\
\text { rapid change in } \\
\text { traditional behaviours }\end{array}$ \\
\hline
\end{tabular}


Table 1 Summary of included papers (Continued)

\begin{tabular}{|c|c|c|c|c|c|c|c|}
\hline Effectiveness of he & ealth education & as an interve & tion designed to pre & vent female & ital mutilation/ & tting (FGM/C): A system & atic review. \\
\hline $\begin{array}{l}\text { Jacoby SD, } \\
\text { Lucarelli M, } \\
\text { Musse F, } \\
\text { Krishnamurthy } \\
\text { A, Salyers V } \\
\text { (2015) }\end{array}$ & $\begin{array}{l}\text { Lewiston, } \\
\text { Maine } \\
\text { United } \\
\text { States. } \\
\text { FGM/C Type } \\
\text { I - IV }\end{array}$ & $\begin{array}{l}\text { Somali } \\
\text { Women, } \\
\text { Individuals } \\
\text { who had } \\
\text { experienced } \\
\text { perinatal } \\
\text { health care }\end{array}$ & $\begin{array}{l}\text { Somali women } \\
\text { Living in Lewiston, } \\
\text { Maine } \\
\text { Participants were } \\
\text { from countries } \\
\text { where FGM/C is } \\
\text { practiced } \\
12 \text { to } 60 \text { years }\end{array}$ & $\begin{array}{l}\text { Mixed- } \\
\text { methods }\end{array}$ & $\begin{array}{l}\text { Interviews } \\
75 \%\end{array}$ & $\begin{array}{l}\text { General information } \\
\text { about women's health } \\
\text { including FGM/C }\end{array}$ & $\begin{array}{l}\text { No participant had } \\
\text { adequate health } \\
\text { literacy } \\
\text { Historietas were } \\
\text { unanimously approved } \\
\text { As appropriate health } \\
\text { education tools }\end{array}$ \\
\hline $\begin{array}{l}\text { Mounir G, } \\
\text { Nehad HM, } \\
\text { Ibtsam MF. } \\
\text { (2003) }\end{array}$ & $\begin{array}{l}\text { Alexandria } \\
\text { University, } \\
\text { Egypt }\end{array}$ & $\begin{array}{l}\text { Female } \\
\text { students } \\
\text { Egypt- } \\
\text { Middle East }\end{array}$ & $\begin{array}{l}\text { Students from } \\
\text { Alexandria } \\
\text { University second } \\
\text { grade } \\
\text { Participants belong } \\
\text { to community } \\
\text { affected by FGM } \\
\text { Mean-19.35 }\end{array}$ & $\begin{array}{l}\text { Quasi- } \\
\text { experiment } \\
\text { El-Shatby } \\
\text { hostel was } \\
\text { the control } \\
\text { group that } \\
\text { did not } \\
\text { receive the } \\
\text { program }\end{array}$ & $\begin{array}{l}\text { Questionnaire } \\
75 \%\end{array}$ & $\begin{array}{l}\text { Training on } \\
\text { Importance of } \\
\text { premarital counselling, } \\
\text { family planning, } \\
\text { breastfeeding, sexually } \\
\text { transmitted diseases } \\
\text { Alternative methods } \\
\text { of family planning, } \\
\text { weaning and } \\
\text { importance of } \\
\text { breastfeeding, } \\
\text { importance of } \\
\text { antenatal care, } \\
\text { methods of } \\
\text { prevention of STDs } \\
\text { Experience and } \\
\text { precautions against } \\
\text { FGM and early } \\
\text { marriage, social } \\
\text { pressure on early } \\
\text { marriage and FGM }\end{array}$ & $\begin{array}{l}\text { Statistically significant } \\
\text { improvement in each } \\
\text { domain of knowledge } \\
\text { measured in } \\
\text { intervention group and } \\
\text { no absolute change } \\
\text { was detected in the } \\
\text { control group } \\
33.3 \% \text { gain scores was } \\
\text { detected for } \\
\text { knowledge about the } \\
\text { term RH and FGM } \\
\text { In regards to effects of } \\
\text { intervention program, } \\
\text { those of high social } \\
\text { class had a higher } \\
\text { post-test score } \\
\text { The program resulted } \\
\text { to significant } \\
\text { improvement in most } \\
\text { of knowledge items } \\
\text { and a shift towards a } \\
\text { positive attitude }\end{array}$ \\
\hline $\begin{array}{l}\text { Olaitan LO } \\
\text { (2010) }\end{array}$ & $\begin{array}{l}3 \text { State } \\
\text { Capitals in } \\
\text { South west } \\
\text { Nigeria }\end{array}$ & $\begin{array}{l}\text { Males and } \\
\text { females } \\
\text { African- } \\
\text { Nigeria } \\
\text { (Yoruba, } \\
\text { Fulani, } \\
\text { Hausa and } \\
\text { Nupe) }\end{array}$ & $\begin{array}{l}\text { Parents } \\
\text { Participants belong } \\
\text { to communities } \\
\text { affected by FGC } \\
15 \text { to } 65 \text { and } \\
\text { above }\end{array}$ & $\begin{array}{l}\text { Survey } \\
\text { No } \\
\text { comparison } \\
\text { group }\end{array}$ & $\begin{array}{l}\text { Questionnaire } \\
75 \%\end{array}$ & $\begin{array}{l}\text { General knowledge } \\
\text { about FGM }\end{array}$ & $\begin{array}{l}\text { No significance difference } \\
\text { existed between males } \\
\text { and females in the } \\
\text { knowledge about FGMC } \\
\text { There was significant } \\
\text { difference based on } \\
\text { age in knowledge } \\
\text { about FGM/C } \\
\text { There was significant } \\
\text { difference based on } \\
\text { educational status } \\
\text { Community health } \\
\text { education is the best } \\
\text { means of providing } \\
\text { health information and } \\
\text { education to people at } \\
\text { every level. }\end{array}$ \\
\hline $\begin{array}{l}\text { Ruiz Jl, Martinez } \\
\text { AP, Bravo PMDM. } \\
\text { (2015) }\end{array}$ & $\begin{array}{l}\text { Spain-Murcia } \\
\text { and } \\
\text { Eastern } \\
\text { Morocco }\end{array}$ & $\begin{array}{l}\text { Males } \\
\text { African- } \\
\text { Living in } \\
\text { Spain and }\end{array}$ & $\begin{array}{l}\text { Male, living in Spain } \\
\text { and Morocco } \\
\text { originally from } \\
\text { countries where } \\
\text { FGM is performed } \\
\text { Participants lived } \\
\text { at least } 18 \text { years in } \\
\text { their countries of } \\
\text { origin and have } \\
\text { personally being in } \\
\text { contact with } \\
\text { women with FGM } \\
\text { Participants } \\
\text { Comprehend } \\
\text { Spanish or French } \\
\text { Between } 20 \text { and } \\
53 \text { years }\end{array}$ & Qualitative & $\begin{array}{l}\text { Semi- } \\
\text { structured } \\
\text { interview } \\
75 \%\end{array}$ & $\begin{array}{l}\text { First-hand knowledge } \\
\text { of the practice and its } \\
\text { foundations-from vari- } \\
\text { ous sensitisation and } \\
\text { personal experience }\end{array}$ & $\begin{array}{l}\text { Sensitised men can } \\
\text { change viewpoints } \\
\text { regarding the practice } \\
\text { Important to use visual } \\
\text { and communication } \\
\text { media in health } \\
\text { education programmes } \\
\text { There is need for new } \\
\text { development of health } \\
\text { education } \\
\text { programmes. }\end{array}$ \\
\hline
\end{tabular}


language. Jacoby et al. found that the use of a 'cultural broker,' who is a translator, helped in mitigating some of the language challenges by translating for both the facilitators and participants in languages they could understand [32].

\section{Gender}

Two studies reported that gender differences of the recipients affected levels of participating in health education interventions $[28,31]$. For example, in the study by Babalola et al. [28], the measure of programme exposure where the radio was the major source revealed that 67. $1 \%$ of men were exposed to at least one component of the programme, from which they learnt more about FGM/C, compared to $61.4 \%$ of women. Diop and Askew [31] in their report on evaluating the effectiveness of education offered by the 'Tostan programme' in Senegal, revealed that there were gender differences in awareness of at least two consequences of FGM/C. Among men, awareness increased from $11 \%$ to $80 \%$ and among women, from $7 \%$ to $83 \%$. The slight difference between the genders was attributed to women's personal experiences of undergoing FGM/C which facilitated a better understanding of the topics.

\section{Marital status}

Three studies reported that some married participants, both males and females, did not benefit from health education interventions. This is because they held on to their belief that FGM/C is a means of controlling promiscuity of girls and women which was a virtue in these communities [31, 35, 38]. However, although FGM/C traditionally was believed to be a prerequisite for marriage, some studies reported that most unmarried participants did not think it was important and they appreciated the health education interventions. The authors concluded that this reflected a natural decline in the practice due to generational differences [30, 33]. Chege et al. [30] on a different note, did not find significant association between marital status and support for or opposition of $\mathrm{FGM} / \mathrm{C}$ and therefore concluded that being married did not act as a barrier to effective health education interventions.

\section{Residential status}

The residential status of intervention groups influenced the effectiveness of health education interventions, especially for participants who were immigrants. Jacoby et al. [32] indicated that refugees in the USA shared common concerns in health care matters. These included general health literacy levels and knowledge of the health implications of FGM/C. Additionally, Chege et al. [30] reported that there were higher chances of encountering more resistance to the intervention from immigrants due to forced law enforcement against FGM/C in the host country.

\section{Socioeconomic factors}

Two socioeconomic factors emerged from the included studies; education and occupation/roles in communities.

\section{Education}

Five studies reflected on the extent to which levels of basic education impacted on access to and acceptability of FGM/C health education programmes. AsekunOlarinmoye and Amusan [29] reported that education levels of the participants determined the acceptability and effectiveness of health education. This manifested in the attitudes expressed by those with no formal education who were more likely to encourage FGM/C. Olaitan [36] similarly reported that there was a significant difference in knowledge about FGM/C based on educational status. Those with more years of education had greater knowledge influenced by health education interventions. Alo and Gbadebo [39] also highlighted that parents with post-secondary education were less inclined to have their daughters undergo FGM/C because they were more likely to be exposed to health education interventions about the practice. They reported that $48 \%$ of those with post-secondary education had none of their daughters cut, compared to $20 \%$ of respondents with no formal education. Mounir et al. [33] reported that, students from higher income families had better improvement in knowledge about FGM/C because their family educational background supported acquisition of such knowledge. Slightly contradictorily, Allam et al. [34] found a considerable amount of ignorance concerning FGM/C existed among the educated population in Egypt, including some doctors and midwives.

\section{Occupation/roles in communities}

The studies by Asekun-Olarinmoye and Amusan [29] and Alo and Gbadebo [39] highlighted that traditional excisors, health professionals, community leaders and religious leaders were not only recipients of health education interventions, but also implementers and change agents for better outcomes of interventions. When such influential individuals refrain from supporting antiFGM/C messages, it can negatively affect the success of health education interventions[30].

\section{Traditions and beliefs Religion}

One study concluded that religious belief was not associated with encouraging the continuation of $\mathrm{FGM} / \mathrm{C}$ and therefore, not a hindrance to effective health education intervention [29]. However, Allam et al. [34] and Chege et 


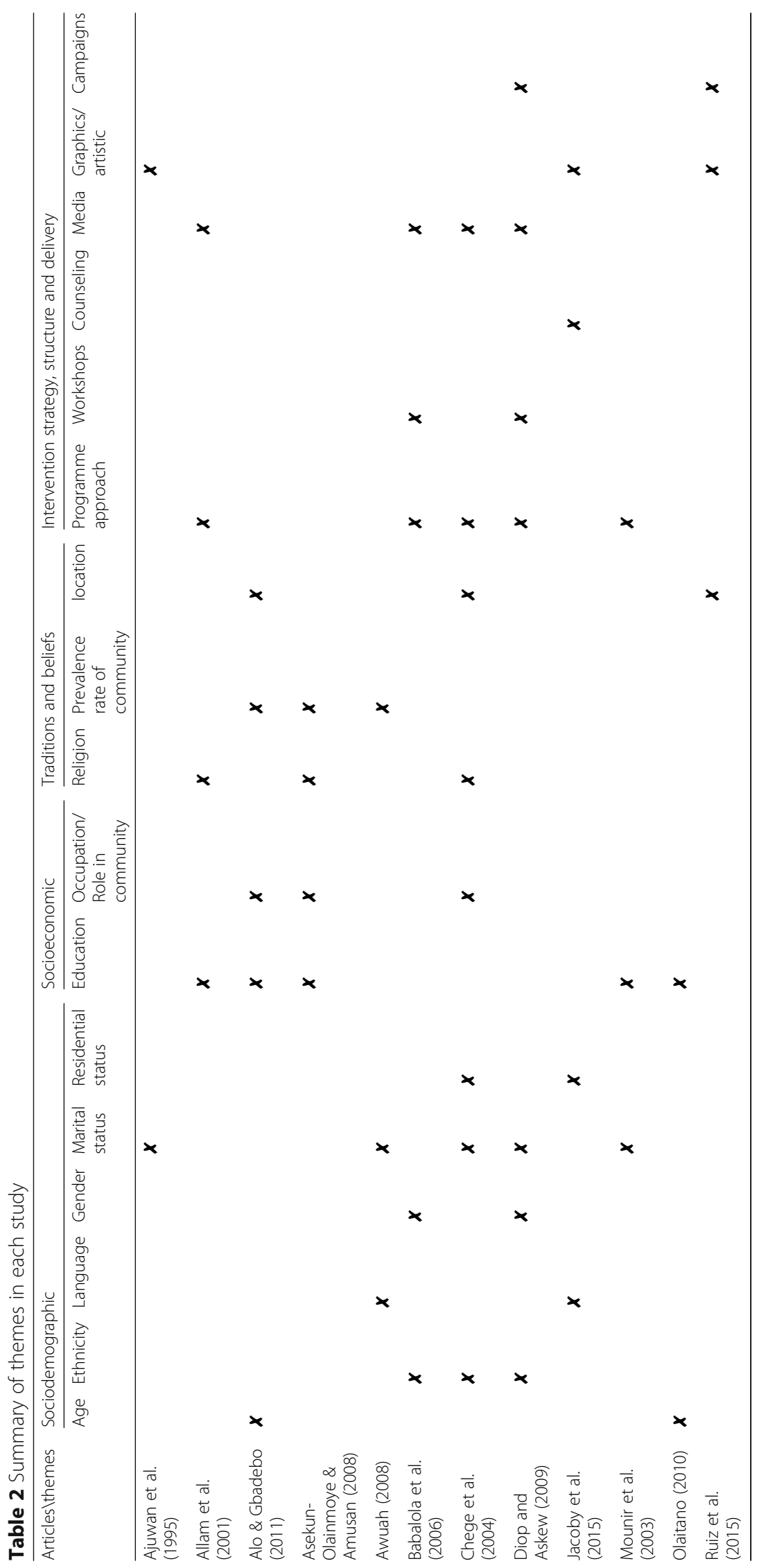


al. [30] reported that religious affiliations of either the participants or the facilitators of health education interventions were likely to affect the effectiveness of the intervention. Distrust predominantly arose when programme facilitators affiliated themselves with a different religion from that of the participants. They also found that involving religious leaders in the health education programmes either positively influenced communities through their teachings about FGM/C, which encouraged abandonment of the practice, or negatively promoted the practice by for instance, referring to it as a religious requirement. Allam et al. [34] emphasised that it was more difficult to educate participants who believed FGM/C is a religious requirement for any faith, because they were more likely to condone the practice.

\section{Prevalence of FGM/C in communities}

Prevalence rates were presented to have an effect on health education interventions. For example, in the study from Ghana by Awuah [35], 100\% $(n=70)$ of the respondents claimed that FGM/C was practiced, an exercise of which about $43 \%$ did not regret. Asekun-Olainmoye and Amusan [29] similarly indicated that $88 \%(n=211)$ of female respondents reported to have had FGM/C, of whom $85 \%$ had no regrets. Alo and Gbadebo [39] reported on differences in prevalence of FGM/C between generations. They observed that attitude and prevalence more often than not go together. This was reflected in the insignificant $4 \%$ intergenerational difference that indicated minimal changes in attitudes following health education interventions that did not justify the efforts made to prevent the practice.

\section{Locality}

Three studies demonstrated the impact that locality has on the effectiveness of the intervention [30, 39]. Chege et al. [30], found that the percentage exposed to anti-FGM/C messages in Ethiopia, among the intervention group, increased from $21 \%$ to $71 \%$ while in Kenya, it increased from $40 \%$ to $59 \%$. The difference was attributed to disparities in societal structures. For example, laws against FGM/C were harsher in Kenya, and their enforcement made it harder for interventions to win trust from FGM/C affected communities. Alo and Gbadebo [39] emphasised that, females in a rural setting were more likely to support FGM/C compared to those living in urbanised communities. They concluded that health education interventions in the rural areas require more intense planning and implementation than the urban areas. Ruiz et al. [37] in the same vein reported that awareness efforts in rural areas needed to be more intense in comparison with urban areas because the inherent isolation in rural areas propagated FGM/C.

\section{Programme strategy, structure and delivery Programme approach}

Methods that health education programmes used to approach the intervention groups were associated with the effectiveness of the interventions. Chege et al. [30], Mounir et al. [33] and Allam et al. [34] reported that it was necessary for health education facilitators to approach communities with caution; otherwise, they would face rejection. In studies that included interviewing as a process of health education, interventions were more effective when males interviewed males and females interviewed females [34]. Diop and Askew noted that researchers needed to integrate with community interviewers, who belonged to the target communities, prior to implementation so as to increase reliability of respondents reports [31].

Four studies showed that the levels of programme exposure impacted on the effectiveness of the health education intervention [28, 30,31,34]. Lower levels of exposure translated to a smaller increase in positive attitude and intended behaviour [30], while higher levels of exposure translated to powerful, effective means for facilitating rapid communal changes [31].

\section{Workshops}

The studies by Babalola et al. [28] and Diop and Askew [31] reported that there was inconsistency in lectures and workshops attendance. This influenced the effectiveness of the health education interventions as it translated to suboptimal outcomes because they yielded insignificant results.

\section{Counselling}

Jacoby et al. [32] showed that counselling early in the antepartum period was more effective than late counselling. This was preferable to the participants on the basis that early intervention gives ample time for thinking and discussing health concerns with spouses.

\section{Media}

Media seemed to be a vital tool for delivering FGM/C health education interventions. Radio appeared to be an effective means to reach the men in most populations and was mostly favourable among young people $[28,31$, 34]. However, in the study by Chege et al. [30], media was disadvantageous because some messages were not always in support of $\mathrm{FGM} / \mathrm{C}$ prevention. This in turn limited the effectiveness of the health education intervention.

\section{Graphics/artistic}

The use of graphics or artistic modes of dissemination mostly enhanced the effectiveness of health education interventions. Jacoby et al. [32] in their study about immigrant Somali women's health literacy and perinatal 
experiences found that Historietas (graphical booklets) were endorsed by participants because they understood the contents better [32]. Practical training was reported by participants as a preference and was endorsed as a means of effective communication of anti-FGM/C including to traditional excisors [37, 38].

\section{Discussion}

This review aimed to assess the effectiveness of health education interventions in preventing FGM/C in the affected communities. We managed to unveil factors that facilitate or hinder effectiveness of health education interventions. Various studies, including systematic reviews, have evaluated different interventions as well as their benefits and effectiveness in preventing FGM/C. Health education, among other interventions, has been regarded as important in contributing to raising awareness about FGM/C, leading to changed attitudes and behaviours in various communities [40, 41].

Our study challenges the approach applied by previous reviews about the effectiveness of FGM/C interventions in general. We ventured to explore health education as an individual intervention, while focusing on the issues that are specific to this particular intervention. Our results show that the effectiveness of FGM/C health education interventions depended on factors linked to sociodemographic factors; socioeconomic factors; traditions and beliefs; and intervention strategy, structure and delivery. The most pronounced finding was that these factors are guaranteed to disturb the process of implementing change through health education interventions. It is therefore, important to ensure that health education interventions have tailored information, communication and education to fit the target population based on their needs. This requires prior understanding of individual capacity and existing knowledge including individual ages and levels of education $[29,30,33,35$, $36,37,38,39]$. The importance of community-based approaches for FGM/C health education interventions cannot be underestimated. The value of this approach is demonstrated by Chege et al. [30] who reported how religious leaders and other key leaders in the communities were used for advocacy against FGM/C. Community leaders are valued individuals and their inclusion in interventions has been recognised to enhance effectiveness particularly where messages relate to sensitive health problems such as HIV [42]. There are a number of strategies to support the process. For example, Mounir et al. [33] described how intervention facilitators in their study dressed in a similar style of clothing to participants in an attempt to encourage shared identity and break down barriers.

It is important to acknowledge that the studies included in this review focused on different communities who may have varying reasons for performing FGM/C, even when they come from the same country. This supports the importance of tailoring interventions to the target population and minimising generalisation.

Personal beliefs and views have been highlighted in a number of contexts to affect attempts to induce change of negative social behaviours because they are highly influenced by prior knowledge, experience and psychological state [43, 44]. An understanding of individual viewpoints and attitude can predict behaviour change [45]. This review has shown that educational background, rate of prevalence of $\mathrm{FGM} / \mathrm{C}$, religion and media all influence the effectiveness of health education interventions $[28,29,30,31,34,35,39]$. Diop and Askew [31], for example contended that there was a reduction in the number of daughters who were cut after their mothers participated in a programme aimed at changing perceptions of FGM/C. Alo [39], however, reported that women's decisions did not matter as their husbands had control over decision-making, influencing their behaviour. This indicated that though the women were aware of the health issues, FGM/C would still be practised. Michie et al. [46] similarly suggested that behaviour change is dependent on psychological capabilities such as strength and skill that could affect individual perception and social opportunities such as cultural norms that could influence behaviour, regardless of individual perception.

While changed attitude and behaviour by individuals is essential, sharing acquired information and change is as important for better results of collective prevention of FGM/C [30]. The willingness to share information however, is dependent on factors such as commitment, enjoyment of helping others, reputation and organisational reward [47]. Diop and Askew [31] for example, indicated that women who participated in an FGM/C programme were encouraged to "adopt" a friend/relative and share information regarding their learning during classes and this proved effective. The programme encouraged sharing of information by establishing community management committees to strengthen village ownership of the programme. However, it is not always a guarantee that group prevention is attained, especially if pro-FGM/C messages are shared instead of messages against FGM/ C. When correct information is communicated and shared effectively, it eventually results in collective knowledge and awareness that in turn influences communal change. This can culminate in wider results for public campaigns and denouncement of the practice [30].

This review acknowledges the factor of acculturation, which has the capacity to influence the attitude and views of immigrant communities, depending on the economic status and legislative changes of the home or host 
countries [48]. Two studies that were conducted in Spain and USA focused on communities with a history of FGM/C living in these high income countries. They found that participants' views and opinions may not be entirely free from acculturation [32, 37]. In addition, the impact of acculturation may not be reflected differently when the host country is a low-middle income country [30] .

According to the $\mathrm{WHO}$, health education presents to communities a package comprising opportunities for learning that are based on sound theories to offer health information. From some perspectives, the FGM/C interventions can be perceived as a top-down approach, with communities being the recipients[19]. With this in mind, Babalola et al. (28) emphasises the importance of integration with communities prior to implementation of interventions. They argued that this increases community acceptance of an intervention, leading to its success.

Findings from this review highlight health education as a promising intervention in preventing FGM/C. The intervention is favoured over other interventions such as legislation, because it is less repressive. Although the law reduces the rate of $\mathrm{FGM} / \mathrm{C}$, it has also been found to drive the practice underground. In other instances, the law has led to parents subjecting girls to $\mathrm{FGM} / \mathrm{C}$ at a younger age before they are susceptible to anti-FGM/C messages. There is also an association between law enforcement and increased medicalisation as well as reduced reporting of FGM/C cases [37, 49-54].

Contextualising health education interventions is only possible when there is sufficient consideration of the characteristics of target populations. Contextualising involves inclusion of the communities in planning the programmes, for instance, involving permanent residents who belong to the target communities as facilitators or research assistants [29]. Religious and other key leaders can also help to promote the interventions [30]. Community members can be involved in dissemination of information to relatives and friends, therefore, encouraging public awareness and resistance to FGM/C [31]. It is evident from this review that tailoring information to fit the needs of the target populations is crucial because it increases the acceptability of the programme and influences quicker dissemination of information among communities.

Despite the strengths, this review was limited because some studies did not indicate the duration of the interventions. It is therefore important to note that based on the nature of the intervention, shorter durations may have offered less chance for programmes to attain desired goals, especially in sharing of information. This review also considered only studies reported in English. Other languages could have reported the issue differently for instance, studies from non-English speaking high-income countries. The review considered only studies that focused on affected communities and excluded studies from non-practicing communities which future research should consider to include.

There is ample room to improve women and girls' safety from FGM/C. Rational approaches through health education interventions should be carefully planned. As Abdulcadir et al. [55] points out, there is a dearth of research focusing on interventions to prevent FGM/C. This includes health education offered by health professionals who work with communities affected by FGM/C. Further research is needed to establish the effectiveness of health education interventions offered to different populations living in high-income countries. Cultural competency especially in the healthcare system can help improve health outcomes and quality of care [56]. Further research therefore is needed to increase the understanding of how best to involve different demographic groups including non-practicing communities, in health education interventions, in order to maximise effective prevention of FGM/C.

\section{Conclusion}

Health education is an important intervention which has the capacity to change deeply engraved beliefs and attitudes attributed to certain health problems such as $\mathrm{FGM} / \mathrm{C}$. When the intervention is comprehensively planned, implemented and evaluated, it can be successful in preventing FGM/C in any target group. This study contributes to the understanding of the facilitators and barriers of effective health education interventions in preventing $\mathrm{FGM} / \mathrm{C}$. Our findings suggest that health education interventions have the potential to influence communal change, which eventually leads to sustainable prevention of FGM/C. The success of health education interventions is dependent on sociodemographic elements, socioeconomic factors, traditions and beliefs and programme approach. Evidence suggests that these factors are vital and require intensive consideration at every stage of the intervention. This ensures increased possibility of influencing communal change in behaviour and attitude, leading to sustainable prevention of FGM/C, thus, improved reproductive health and wellbeing of individuals and communities.

\section{Additional file}

Additional file 1: Search strategies. (DOCX $12 \mathrm{~kb}$ )

\section{Abbreviations}

CBJ: Caroline Bradbury-Jones; FGM/C: Female genital mutilation/cutting; HS: Harpreet Sihre; JT: Julie Taylor; LD: Lucy Doos; SW: Susan Waigwa; UK: United Kingdom; USA: United States of America; WHO: World Health Organization 


\section{Acknowledgements}

Harpreet Sihre (HS) checked the screening of the identified papers and the extracted data for accuracy.

\section{Funding}

This study was not directly funded by any external body.

\section{Availability of data and materials}

Interested parties can obtain all available data by contacting the corresponding author.

\section{Authors' contributions}

SW undertook the systematic review, carried out the thematic synthesis and wrote the manuscript. LD, CBJ and JT participated in the design of the systematic review, advised in the thematic synthesis and revised the manuscript. All authors agreed the final version of the manuscript.

\section{Ethical approval and consent to participate}

Not applicable.

\section{Competing interests}

The authors declare that they have no competing interests.

\section{Publisher's Note}

Springer Nature remains neutral with regard to jurisdictional claims in published maps and institutional affiliations.

\section{Author details}

${ }^{1}$ School of Nursing, College of Medical and Dental Science, University of Birmingham, Edgbaston, Birmingham B15 2TT, UK. ${ }^{2}$ School of Social policy, College of Social Sciences, University of Birmingham, Birmingham, UK. ${ }^{3}$ School of Nursing, College of Medical and Dental Sciences, University of Birmingham and Birmingham Women's and Children's Hospital NHS Foundation Trust, Birmingham, UK

\section{Received: 28 October 2017 Accepted: 2 April 2018}

\section{Published online: 12 April 2018}

\section{References}

1. UNICEF. Female Genital Mutilation/Cutting: A statistical overview and exploration of the dynamics of change. New York: UNICEF; 2013.

2. UNICEF: Female genital mutilation: current status and progress. 2016. http:// data.unicef.org/child-protection/fgmc.html. Accessed 25 Apr 2016.

3. Khosla R, Banerjee J, Chou D, Say L, Fried ST. Gender equality and human rights approaches to female genital mutilation: a review of international human rights norms and standards. Reprod Health. 2017;14(1):59.

4. Ceschia A. FGM: the mutilation of girls and young women must stop. Lancet. 2015;385(9967):483-4.

5. Maurice J, Khanna J. WHO: Female genital mutilation: New knowledge spurs optimism. World Health Organisation; 2006.

6. Goldberg H, Stupp P, Okoroh E, Besera G, Goodman D, Danel I. Female genital mutilation/cutting in the United States: Updated estimates of women and girls at risk, 2012. Public Health Rep. 2016;131(2):340-7.

7. Macfarlane A, Dorkenoo E. Prevalence of female genital mutilation in England and Wales: National and local estimates. 2015.

8. Berg RC, Denison EM-L, Fretheim A: Psychological, social and sexual consequences of female genital mutilation/cutting (FGM/C): a systematic review of quantitative studies. 2010

9. Berg RC, Underland V, Odgaard-Jensen J, Fretheim A, Vist GE. Effects of female genital cutting on physical health outcomes: a systematic review and meta-analysis. BMJ open. 2014;4(11):e006316.

10. Mwanri L, Gatwiri GJ. Injured bodies, damaged lives: experiences and narratives of Kenyan women with obstetric fistula and Female Genital Mutilation/Cutting. Reprod Health. 2017;14(1):38.

11. WHO study group on female genital mutilation and obstetric outcome, Banks E, Meirik O, Farley T, Akande O, et al. Female genital mutilation and obstetric outcome: WHO collaborative prospective study in six African countries. Lancet. 2006;367(9525):1835-41.

12. HMGovernment. Female genital mutilation: Multi agency practice guidelines. Crown; 2011.
13. Edouard E, Olatunbosun O, Edouard L. International efforts on abandoning female genital mutilation. Afr J Urol. 2013;19(3):150-3.

14. WHO, UNICEF: Female genital mutilation: a joint WHO/UNICEF/UNFPA statement: World Health Organization; 1997.

15. Berg RC, Denison EM-L, Fretheim A: Factors promoting and hindering the practice of female genital mutilation/cutting (FGM/C). 2010.

16. ORCHID: Country profiles. 2016. https://orchidproject.org/country-profiles/. Accessed 17 Apr 2016

17. Mostafa S, El Zeiny N, Tayel S, Moubarak E. What do medical students in Alexandria know about female genital mutilation? 2006. http://apps.who.int/ iris/bitstream/handle/10665/117197/12_S2_2006_78_92.pdf?sequence= 1 \&isAllowed=y.

18. Berg RC, Odgaard-Jensen J, Fretheim A, Underland V, Vist G. An updated systematic review and meta-analysis of the obstetric consequences of female genital mutilation/cutting. Obstet Gynecol Int. 2014;2014:542859.

19. WHO: Health topics: Health education. 2016. http://www.who.int/topics/ health education/en/. Accessed 25 Apr 2016.

20. WHO. Health education: theoretical concepts, effective strategies and core competencies: a foundation document to guide capacity development of health educators. 2012

21. Ghrayeb FA, Rusli AM, Al Rifai A, Ismail IM. The impact of education program on smoking prevention: an intervention study among 16 to 18 years old in Palestine. Pak J Nutr. 2013;12(8):782.

22. Adekunle S. Effects of health education on cigarette smoking habits of young adults in tertiary institutions in a northern Nigerian state. Health Sci J. 2011;5(3).

23. Singh D, Negin J, Orach CG, Cumming R. Supportive supervision for volunteers to deliver reproductive health education: a cluster randomized trial. Reprod Health. 2016;13(1):126.

24. Bornstein MH, Cote LR, Haynes OM, Hahn C-S, Park Y. Parenting Knowledge: Experiential and Sociodemographic Factors in European American Mothers of Young Children. Dev Psychol. 2010;46(6):1677-93.

25. Richard F, Ahmed W, Denholm N, Dawson A, Varol N, Essén B, Johnsdotter S, Bukuluki P, Ahmed W, Naeema AGH, et al. Female Genital Mutilation/ Cutting: sharing data and experiences to accelerate eradication and improve care: part 2. Reprod Health. 2017;14(2):115.

26. Pluye $P$, et al. Mixed Methods Apparaisal Tool (MMAT). 2011. http:// mixedmethodsappraisaltoolpublic.pbworks.com/w/file/fetch/84371689/ MMAT\%202011\%20criteria\%20and\%20tutoria|\%202011-06-29updated2014. 08.21.pdf. Accessed 16 June 2016

27. Yang L, Sahlqvist S, McMinn A, Griffin SJ, Ogilvie D. Interventions to promote cycling: systematic review. BMJ. 2010;341:c5293.

28. * Babalola S, Brasington A, Agbasimalo A, Helland A, Nwanguma E, Onah N. Impact of a communication programme on female genital cutting in eastern Nigeria. Trop Med Int Health. 2006;11(10):1594-603.

29. * Asekun-Olarinmoye EO, Amusan OA. The impact of health education on attitudes towards female genital mutilation (FGM) in a rural Nigerian community. Eur J Contracept Reprod Health Care. 2008;13(3):289-97.

30. * Chege J, Askew I, Igras S, Mutesh JK. Testing the effectiveness of integrating community-based approaches for encouraging abandonment of female genital cutting into CARE's reproductive health programs in Ethiopia and Kenya. Washington, DC: Population Council; 2004

31. * Diop NJ, Askew I. The effectiveness of a community-based education program on abandoning female genital mutilation cutting in Senegal. Stud Fam Plann. 2009;40(4):307-18.

32. * Jacoby S, Lucarelli M, Musse F, Krishnamurthy A, Salyers V. A MixedMethods Study of Immigrant Somali Women's Health Literacy and Perinatal Experiences in Maine. J Midwifery Womens Health. 2015;60(5):593.

33. * Mounir GM, Mahdy NH, Fatohy IM. Impact of health education program about reproductive health on knowledge and attitude of female Alexandria University students. J Egypt Public Health Assoc. 2002;78(5-6):433-66.

34. * Allam MF, de Irala-Estevez J, Navajas RFC, del Castillo AS, Hoashi JS, Pankovich MB, Liceaga JR. Factors associated with the condoning of female genital mutilation among university students. Public Health. 2001:115(5):350-5.

35. * Awuah JB. Female genital mutilation: a study in Aboabo, a suburb of Kumasi, Ghana. West Afr J Nurs. 2008;19(1):26-32. 27p

36. * Olaitan OL. Knowledge of female genital cutting among parents in south west Nigeria. Transl Biomed. 2010;1(2).

37. * Ruiz IJ, Martinez PA, Bravo MDP. Key points for abolishing Female Genital Mutilation from the perspective of the men involved. Midwifery. 2016;34:30-5. 
38. * Ajuwon AJ, Brieger WR, Oladepo O, Adeniyi JD. Indigenous surgical practices in rural southwestern Nigeria: Implications for disease prevention. Health Educ Res. 1995;10(3):379-84.

39. * Alo OA, Gbadebo B. Intergenerational Attitude Changes Regarding Female Genital Cutting in Nigeria. J Womens Health. 2011;20(11):1655-61.

40. Berg RC, Denison EM. A realist synthesis of controlled studies to determine the effectiveness of interventions to prevent genital cutting of girls. Paediatr Int Child Health. 2013;33(4):322-33.

41. Berg RC, Denison E. Effectiveness of interventions designed to prevent female genital mutilation/cutting: a systematic review. Stud Fam Plann. 2012:43(2):135-46.

42. Li AT-W, Wong JP-H, Cain R, Fung KP-L. Engaging African-Caribbean, Asian, and Latino community leaders to address HIV stigma in Toronto. Int J Migr Health Soc Care. 2016;12(4):288-300.

43. Bernstein D. Essentials of psychology: Cengage Learning; 2013.

44. Hendriks H, Bvd P, de Bruijn G-J. Subjective reality: the influence of perceived and objective conversational valence on binge drinking determinants. J Health Commun. 2015;20(7):859-66.

45. Yzer M. The integrative model of behavioral prediction as a tool for designing health messages. Health Commun. 2012:21-40.

46. Michie S, van Stralen MM, West R. The behaviour change wheel: a new method for characterising and designing behaviour change interventions. Implement Sci. 2011;6(1):42.

47. Tan CN-L, Ramayah T. The role of motivators in improving knowledgesharing among academics. Inf Res. 2014;19(1):n1.

48. Jass G, Massey DS. Immigrant health: selectivity and acculturation. In: IFS Working Papers, Institute for Fiscal Studies (IFS); 2004.

49. Boyle EH, Corl AC. Law and culture in a global context: Interventions to eradicate female genital cutting. Annu Rev Law Soc Sci. 2010;6:195-215.

50. Yoder PS, Abderrahim N, Zhuzhuni A. Female genital cutting in the Demographic and Health Surveys: a critical and comparative analysis. 2004.

51. Easton P, Monkman K, Miles R. Social policy from the bottom up: abandoning FGC in sub-Saharan Africa. Dev Pract. 2003;13(5):445-58.

52. Jackson EF, Akweongo P, Sakeah E, Hodgson A, Asuru R, Phillips JF. Inconsistent reporting of female genital cutting status in northern Ghana: Explanatory factors and analytical consequences. Stud Fam Plann. 2003;34(3):200-10.

53. Ahlberg BM, Krantz I, Lindmark G, Warsame M. 'It's only a tradition': making sense of eradication interventions and the persistence of female 'circumcision' within a Swedish context. Crit Soc Policy. 2004;24(1):50-78.

54. Yount KM, Carrera JS. Female genital cutting and reproductive experience in Minya, Egypt. Med Anthropol Q. 2006;20(2):182-211.

55. Abdulcadir J, Say L, Pallitto C. What do we know about assessing healthcare students and professionals' knowledge, attitude and practice regarding female genital mutilation? A systematic review. Reprod Health. 2017;14(1):64.

56. Ihara E. Cultural Competence in Health Care: Is it Important for People with Chronic Conditions? Center on an Aging Society; 2004

\section{Ready to submit your research? Choose BMC and benefit from:}

- fast, convenient online submission

- thorough peer review by experienced researchers in your field

- rapid publication on acceptance

- support for research data, including large and complex data types

- gold Open Access which fosters wider collaboration and increased citations

- maximum visibility for your research: over $100 \mathrm{M}$ website views per year

At BMC, research is always in progress.

Learn more biomedcentral.com/submissions 